\title{
Optimizing multi-graph learning based salient object detection
}

\author{
Shiqi Li ${ }^{\mathrm{a}}$, Cheng Zeng ${ }^{\mathrm{a}, *}$, Yan $\mathrm{Fu}^{\mathrm{a}}$, Shiping $\mathrm{Liu}^{\mathrm{a}}$ \\ ${ }^{a}$ School of Mechanical Science and Engineering, Huazhong University of Science and Technology, Wuhan, 430074, China
}

\begin{abstract}
In this paper, we propose a novel bottom-up saliency detection algorithm to effectively detect salient objects. Different from most existing methods that are not robust to complex scenes, we utilize multi-graph learning to take various scenes into consideration. First, multiple features are used to represent superpixels, and then measured by multiple distance metrics to construct multiple graphs. The motivation is to take advantage of their complementary information to cope with different environments. Second, fixation and boundary cues are respectively used as foreground and background seeds. The fixation is effective for crowded backgrounds because of the observation that regions within eye fixations are very likely the image foreground. Third, we integrate the multiple graphs and seeds into a regularized and optimized multi-graph based learning framework to effectively generate foreground-based and background-based saliency maps. Finally, we integrate these two saliency maps to obtain a more smooth and accurate saliency map. Extensive experiments are conducted on five benchmark datasets. Experimental results show that the proposed bottom-up saliency detection method yields comparable or better results against the state-of-the-art methods, and is robust to both cluttered and clean scenes.
\end{abstract}

Keywords: Salient object detection, Multi-graph learning, Superpixel, Fixation and boundary cues.

\section{Introduction}

The goal of salient object detection is to detect the most salient regions and then segment entire salient objects out. It has recently attracted more attention in computer vision community as it can be used as an important preprocessing step before further processing. Consequently, the applications of salient object detection have flourished in numerous areas, including recognition [1], [2], image retrieval [3], image retargeting [4], GPS location estimation [5], and human-robot interaction [6], [7]. In general, saliency detection methods can be divided into two categories, i.e., bottom-up and top-down. Bottom-up methods are data-driven, while top-down methods are goal-driven. Compared with top-down models, bottom-up models have attracted more interest from researchers due to their simple mechanisms. In this paper, we focus on the bottom-up salient object detection tasks.

In the past decades, a lot of bottom-up saliency detection approaches have been proposed for detecting salient objects in images. Itti et al. [8] proposed one of the earliest models. Their model combined bottom-up visual features of color, intensity, and orientation based on center-surround mechanisms to construct a saliency map. Liu et al. [9] integrated a set of novel features with a conditional random field learning to generate a binary saliency result. Achanta et al. [10] performed saliency estimation by using the color contrast of each pixel in the entire image. These bottomup methods use pixel as the basic units, which are time-consuming and easily affected by small-scale noise in an image. A reasonable solution is to exploit the superpixel-based scheme that abstracts the image by clustering pixels with similar properties into perceptually homogeneous regions. Yang et al. [11] used boundary superpixels as seeds to detect salient regions in images through graph-based manifold learning. Wang et al. [12] selected background and foreground seeds successively from compact superpixels, and then integrated background and foreground saliency maps to generate the final saliency map. It is known to be useful for eliminating background noise and reducing computation by treating each superpixel as a processing unit.

\footnotetext{
${ }^{*}$ Corresponding author

Email address: chengzhust@163. com (Cheng Zeng)
} 


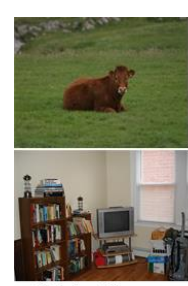

Original image

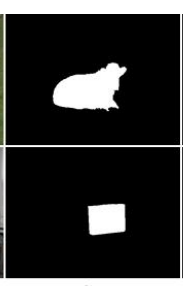

GT

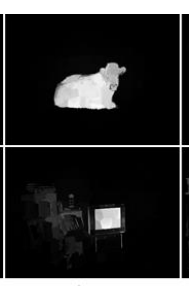

Ours

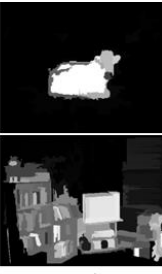

$\mathrm{RC}$

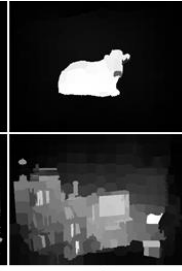

MC

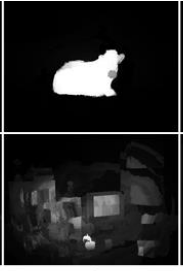

DSR

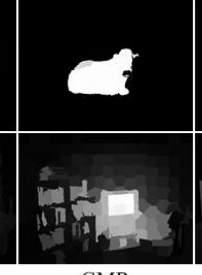

GMR

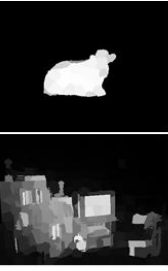

RBD

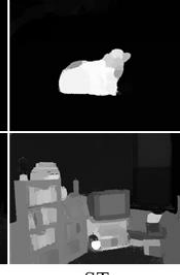

ST

Fig. 1. Examples of salient object detection against state-of-the-art methods (RC [14], MC [15], DSR [16], GMR [11], RBD [17] and ST [18]) in different scenes.

While existing bottom-up saliency methods have demonstrated impressive results, they are still limited to scenes that do not involve complex background. As shown in Fig. 1, when a salient object locates in the low-clutter environment, the state-of-the-art saliency detection methods have impressive performance to obtain saliency maps. However, when a scene contains several objects with crowded background, saliency methods fail to acquire comparable saliency maps compared with ground truth, which is witnessed by the slow progress on complex datasets [13]. One of the main reasons is that bottom-up methods usually lack sufficient priors to measure salient objects in cluttered backgrounds. Another reason lies in the complementarity between the features adopted in those methods are not rich, which leads to the poor robustness in highlighting the salient objects in complex scenes. Finally, some bottom-up methods are computationally expensive as they often need to evaluate the saliency of each pixel.

In this paper, we address these problems with a novel multi-graph based saliency method of exploiting the complementarity of multiple graphs to detect salient objects in cluttered scenes. First, we utilize multiple features of superpixel, including color, color contrast, normal distribution, histogram and edge, to cope with various objects in clutter scenes. In contrast to [11] which only use single distance metric, we measure those features with multiple distance metrics to construct multiple graphs in order to leverage the complementarity between them. Second, we select foreground seeds from fixation cues and background seeds from boundary cues. Compared with other methods [12], [19], the proposed method using fixation seeds are more likely to indicate the salient objects. Third, the background-based and foreground-based saliency maps are respectively generated by ranking the similarity of the superpixels with foreground and background seeds in an optimized multi-graph based framework. Finally, we combine the two saliency maps to obtain the pixel-level saliency map. The pipeline of our method is shown in Fig. 2.

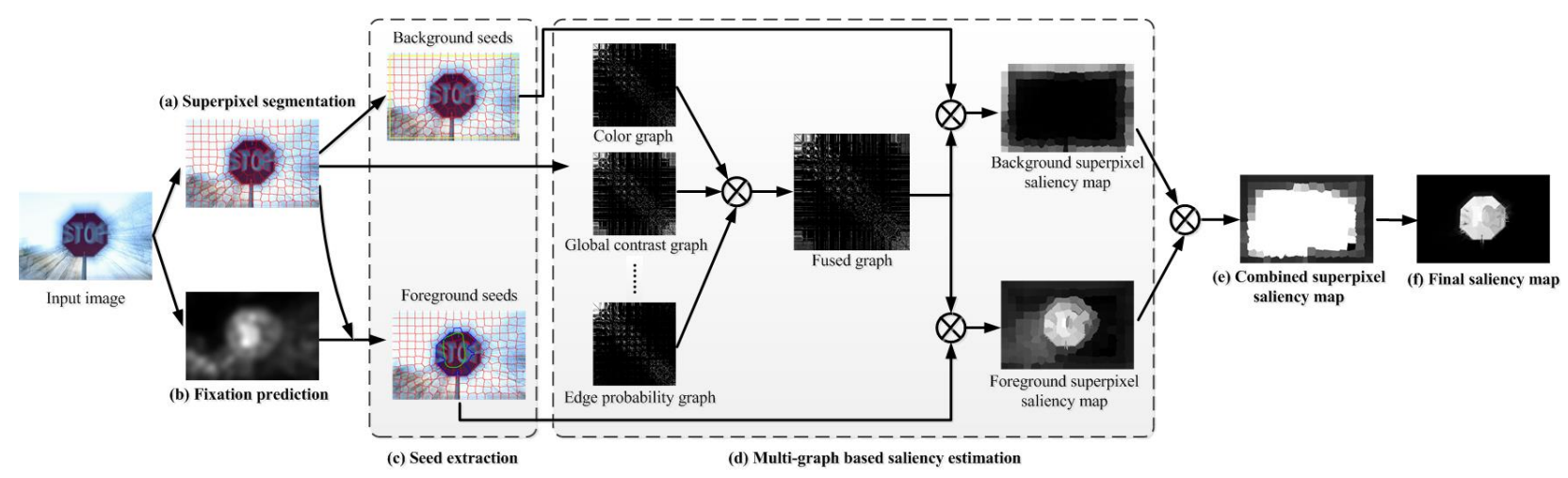

Fig. 2. The pipeline of the proposed method. (a): we adopt a superpixel method to extract the uniform regions from the input image. (b): the location of the salient object is predicted by fixation model. (c): foreground and background seeds are respectively generated by using fixation information and boundary priors. (d): multiple feature graphs and seeds are integrated into a regularized multi-graph based framework to perform saliency estimation and generate the background and foreground superpixel saliency maps. (e): we integrate the two superpixel saliency maps to get the combined superpixel saliency map. (f): we assign the combined superpixel saliency value to each image pixel to obtain the final saliency map. 
To summarize, the main contributions of this paper are threefold:

(1) Instead of only using a single feature or a single distance metric, we measure multiple features by multiple distance metrics to cope with variant scenes.

(2) We introduce the eye fixations as foreground seeds which are more robust to locate salient regions in crowded environments against other saliency detection methods.

(3) We propose an optimized multi-graph based learning algorithm to simultaneously employ multiple features and multiple distance metrics, which optimally leverage the complementary nature of multiple graphs to obtain the more stable and reliable saliency map.

The remainder of the paper is organized as follows: Section 2 reviews related works on salient object detection. Section 3 gives the detailed description of the optimized multi-graph based saliency method. Section 4 presents the extensive experimental results and comparisons. Finally, conclusion and future work are drawn in section 5.

\section{Related works}

This section reviews representative state-of-the-art salient object detection methods. Comprehensive studies about salient object detection methods are presented in [20], [21]. We concentrate on the following aspects that relate to our bottom-up saliency detection approach.

\subsection{Superpixel-based saliency detection}

In general, saliency detection can be divided into two categories regarding to processing units, i.e., pixel-based and superpixel-based. Compared with pixel-based methods, superpixel-based methods are increasingly popular because of the stability and efficiency of superpixels in preserving the structural information of salient objects. Wang et al. [12] first selected background and foreground seeds successively from compact superpixels, and then integrated background and foreground saliency maps to generate the final saliency map. Li et al. [22] used a quadratic programming framework to adaptively optimize the regional saliency values to meet the hypotheses of visual rarity, center-bias, and mutual correlation on a single image. Fu et al. [23] first performed superpixel pre-segmentation and then computed color contrast together with color distribution of superpixels to output saliency map. Kim et al. [24] utilized relative location and color contrast of superpixels to perform local saliency estimation, and combined global saliency estimation of high dimensional color space to generate a saliency map. Zhu et al. [25] segmented an image into multi-size superpixels to represent its fine details as well as large-scale structures. Liu et al. [26] extracted motion and color histograms at superpixel level and frame level as features for spatiotemporal saliency detection in videos, which temporal and spatial saliency maps were generated by using motion distinctiveness estimation and spatial sparsity of superpixels.

\subsection{Usage of distance metric and feature}

Various distance metrics have been employed to measure the saliency of superpixel pairs. The most widely-applied metric is the Minkowski distance family [19], [27], e.g., $L_{2}$ distance metric. Hwang et al. [19] used $L_{2}$ to compute the Euclidean distance between the superpixels in CIELab color space. Fu et al. [27] respectively calculated the CIELab color and intervening contour cue with $L_{2}$ and $L_{\infty}$ distance metrics. In addition to these metrics, several measuring techniques for distribution contrasts such as the KL Divergence [28] and the Wasserstein distance [25] are also used in previous methods to identify local differences. Zhu et al. [25] applied the Wasserstein distance to score contrasts between superpixels in terms of multivariate normal distributions. Fang et al. [29] extracted intensity, color, and texture features from the JPEG bit-steam to calculate the saliency map in the compressed domain. Tian et al. [30] used a learning approach to generate complementary envelope and sketch maps by integrating multiple raw maps generated with several predefined visual saliency models for foreground object segmentation. However, the optimal distance metric varies for different features, and existing methods either use single distance metric or single feature to perform saliency detection, which leads to the poor performance of these methods in complex scenes. Different from these approaches, we choose multiple distance metrics for multiple features to construct multiple graphs in a graph based learning framework, which exploit the complementarity nature of multiple graphs to handle clutter backgrounds for saliency detection. 


\subsection{Graph-based saliency detection}

Given several nodes as queries, the graph based saliency detection is defined to measure the relevance between the remaining nodes and queries. Yang et al. [11] constructed a graph to connect superpixel nodes and estimated the saliency via graph-based manifold ranking with four image boundary nodes separately. Four saliency maps are then multiplied to achieve the final saliency detection. Jiang et al. [15] integrated the background cues into the absorbing Markov chain for saliency. Fu et al. [27] utilized the normalized graph cut to capture color and edge information of image to cluster visual contents. Similar to Yang [11], Qiu et al. [31] used rare shapes from mid-level cues as salient seeds in a graph-based manifold learning framework to generate the saliency map. However, these methods cannot always obtain salient objects in crowded and cluttered backgrounds because the queries of these graphs based methods are not efficient enough to indicate the salient objects. In contrast to these methods, the fixation information we utilized is more robust in these scenes.

It is worth mentioning that the work has been proposed by [32], as it shares the idea of using fixation prediction with ours. In their work, they generate the segmentation of object candidates by a generic object proposal method, and then estimate each candidate with fixations via a scoring function. Instead of first fully segmenting the scene into object candidates and ranking object candidates with fixations, we merely take advantage of fixations to acquire salient regions. Then, we take salient regions as seeds into a regularized and optimized multi-graph based learning framework to effectively generate saliency maps.

\section{Optimized multi-graph learning based saliency method}

This section details the proposed optimized multi-graph learning based saliency detection method. First, the extraction and representation of superpixel are described in section 3.1. We select multiple distance metrics to measure multiple features, which is presented in section 3.2. The multi-graph construction and saliency seeds extraction are discussed in section 3.3 and section 3.4, respectively. The similarity of superpixels with saliency seeds via singlegraph based method is ranked in section 3.5. Section 3.6 introduces the optimized multi-graph based framework to exploit the complementarity of multiple features to handle variant scenes. Finally, we assign the combined superpixel saliency value to each image pixel to acquire pixel-level saliency map, which is stated in section 3.7.

\subsection{Superpixel extraction and representation}

As demonstrated in recent studies, features from superpixels are effective and efficient for salient object detection. In order to extract the uniform regions from an image, we adopt superpixel methods used by many other saliency segmentation models. We test five typical algorithms, including Watersheds [33], Normalized cuts [34], Mean shift [35], Turbopixels [36], and SLIC [37] to generate superpixels. The experiment shows that Watersheds and Mean shift do not allow the number of superpixels and compactness to be controlled, the edge-preserving of Normalized cuts is very poor, Turbopixels produces the most compact and regular superpixel, whereas it suffers from low computational efficiency. We choose the SLIC for superpixels extraction because of its low computational cost and high segmentation performance. For a given image $I$, we over-partition it to build the superpixel set $R=\left\{R_{1}, \ldots, R_{N}\right\}$, and we set the number of superpixels $N=200$ in all the experiments. Fig. 4(a) shows an example of superpixel extraction.

To represent superpixels for saliency detection, we extract multiple priors that are commonly used in saliency detection. Table 1 shows the features that we have used. We first utilize the color prior as it is one of the most widely used cues for saliency methods. Since Lab performs better to simulate human color perception compared with other color spaces (e.g., RGB) [38], we represent color features by averaging pixel color in CIELab color space. Also, we extract edge probability map to represent edge information. It is worth noting that any edge detector that outputs an edge probability map can be employed. We use the edge detector algorithm proposed by [39] as it has state-of-the-art performance and reasonably fast processing speed.

Next we use the global contrast and local contrast as color contrast features [24]. The global contrast of each superpixel $R_{i}$ is measured using the Euclidean distance of color features with all the other superpixels as follows:

$$
G C\left(R_{i}\right)=\sum_{j=1}^{N}\left\|c_{i}-c_{j}\right\|
$$


where $c_{i}$ and $c_{j}$ denote color features of the $i^{\text {th }}$ and $j^{\text {th }}$ superpixels, respectively. We only use the CIELab color channels to calculate the color contrast feature, so it has three channels. The local contrast of the color features is defined as:

$$
\begin{gathered}
L C\left(R_{i}\right)=\sum_{j=1}^{N} \omega_{i, j}^{p}\left\|c_{i}-c_{j}\right\| \\
\omega_{i, j}^{p}=\frac{1}{Z_{i}} \exp \left(-\frac{1}{2 \sigma_{p}^{2}}\left\|p_{i}-p_{j}\right\|\right)
\end{gathered}
$$

where $Z_{i}$ is the normalization term, $\sigma_{p}$ controls the effectiveness of neighboring superpixels, $p_{i}$ and $p_{j}$ denote the normalized central position of the $i^{\text {th }}$ and $j^{\text {th }}$ superpixels, respectively. In our experiments, we set $\sigma_{p}^{2}=0.25$.

We have also utilized local feature statistics inside each superpixel which are summarized by Gaussian distribution so as to capture more information about the superpixels contrasts. The Gaussian distribution is made not only because normality makes further estimations convenient to solve, but also due to its popularity in saliency detection models [25], [40]. For numerical description, we apply maximum likelihood estimation of the normal distribution parameters and obtain mean and variance values as:

$$
\begin{gathered}
\mu\left(R_{i}\right)=\frac{1}{m} \sum_{k=1}^{m} c_{k}=\overline{P^{i}} \\
\sum\left(R_{i}\right)=\frac{1}{m} \sum_{k=1}^{m}\left(c_{k}-\overline{P^{i}}\right)^{2}=\overline{\left(P^{i}\right)^{2}}-{\overline{P^{i}}}^{2}
\end{gathered}
$$

where $m$ is the number of pixels of $R_{i}$, and $c_{k}$ denotes the color feature of $k^{\text {th }}$ pixel. Once the parameters of the multivariate normal distributions have been computed, we obtain the normal distribution for the superpixel $R_{i}$ :

$$
N\left(R_{i}\right)=\left[\mu\left(R_{i}\right), \sum\left(R_{i}\right)\right]
$$

For texture feature, we use the color histogram prior as it is one of the most effective features for saliency detection [41]. Histogram can tolerate noise and minor intra-class differences, and its degree of tolerance can be controlled by choosing appropriate numbers of bins. The histogram feature of the superpixel $R_{i}$ is measured using statistical information of CIELab color space. In addition, in order to eliminate the effect of data magnitudes, we normalize each local histogram for each superpixel so that it sums up to 1 . In the end, the histogram feature is defined as:

$$
H\left(R_{i}\right)=\left[h_{i 1}, h_{i 2}, \ldots, h_{i b}\right], \quad \sum_{k=1}^{b} h_{i k}=1
$$

where $b$ is the number of histogram bins. In our experiments, we use eight bins for each color channel. And Fig. 3 shows an example of all features we used in the paper.

\subsection{Multiple distance metrics selection}

The distance metric determines the similarity measures of superpixel pairs. It plays an important role in many machine learning algorithms for saliency detection. As stated in section 2, existing saliency methods only use single distance metric for all features, which makes it difficult to cope with various scenes. Different from those approaches, we measure features with multiple distance metrics to exploit the complementarity nature of features for saliency detection. Table 1 summarizes the distance metrics for different features that we have used.

(1) $L_{2}$ Distance: The $L_{2}$ distance is the Euclidean measure for contrast between superpixels. It is the most widelyapplied metric for saliency detection methods [11], [27], [31]. The $L_{2}$ distance is written as:

$$
L_{2}\left(R_{i}, R_{j}\right)=\sqrt{\sum_{k=1}^{3}\left(c_{i}^{k}-c_{j}^{k}\right)^{2}}
$$




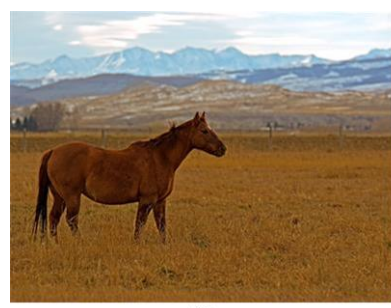

(a)

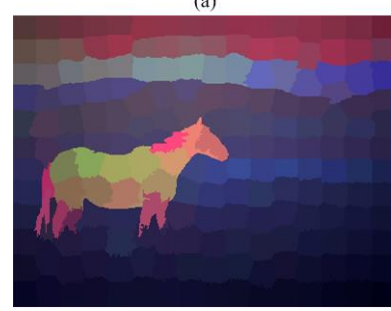

(e)

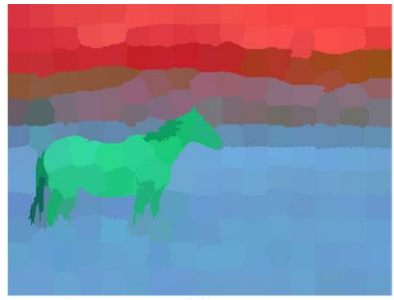

(b)

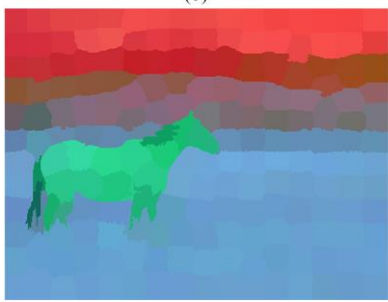

(f)
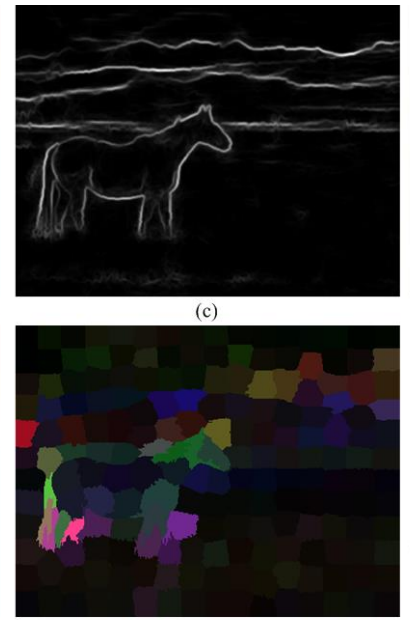

(g)

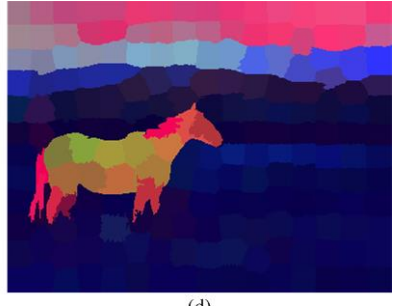

(d)

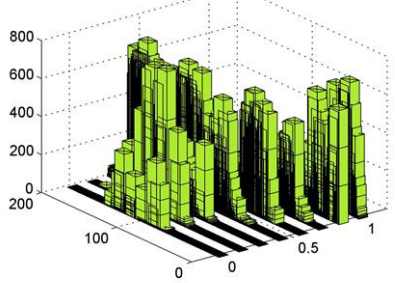

(h)

Fig. 3. Illustration of features we used. (a) original image; (b) the CIELab color feature; (c) the edge probability map feature; (d) the global contrast of the color feature; (e) the local contrast of the color feature; (f) the mean value of Gaussian distribution feature; $(\mathrm{g})$ the variance value of Gaussian distribution feature; $(\mathrm{h})$ the color histogram feature.

where $k$ denotes one of the three channels of CIELab color space. Global contrast and local contrast are also measured by using $L_{2}$ distance.

(2) $\mathcal{W}_{2}$ Distance: The $\mathcal{W}_{2}$ distance (second Wasserstein distance) was first introduced as a measure for centersurround contrast by Klein and Frintrop [36] and achieved reasonable results for saliency detection. It is defined as follows:

$$
\mathcal{W}_{2}\left(R_{i}, R_{j}\right)=\left[\inf _{\gamma \in \Gamma\left(R_{i}, R_{j}\right)} \int_{\mathbb{R} \times \mathbb{R}}|x-y|^{2} \mathrm{~d} \gamma(x, y)\right]^{\frac{1}{2}}
$$

where $\Gamma\left(R_{i}, R_{j}\right)$ denotes the set of all couplings of $R_{i}$ and $R_{j}$. The Wasserstein distance represents the minimum cost of transforming one distribution into another. In the computer vision, the discretized Wasserstein distance is also well known as Earth Movers Distance.

The integral of Eq. (9) is very intractable for arbitrary and continuous distributions, but thankfully can be solved analytically in case of normal distributions. As introduced in [42], an explicit solution for multivariate normal distributions $R_{i} \sim N\left(\mu\left(R_{i}\right), \sum\left(R_{i}\right)\right)$ and $R_{j} \sim N\left(\mu\left(R_{j}\right), \sum\left(R_{j}\right)\right)$ is defined as:

$$
W_{2}\left(R_{i}, R_{j}\right)=\left[\left\|\mu\left(R_{i}\right)-\mu\left(R_{j}\right)\right\|^{2}+\sum R_{i}+\sum R_{j}-2 \sqrt{\sum R_{i} \sum R_{j}}\right]^{\frac{1}{2}}
$$

(3) Hellinger Distance: The Hellinger distance [43] is a measure of difference of two probability distributions with respect to a probability measure $\lambda$, and it is commonly used for histograms. The square of the Hellinger distance is defined as:

$$
H(P, Q)=\frac{1}{2} \int\left(\sqrt{\frac{\mathrm{d} P}{\mathrm{~d} \lambda}}-\sqrt{\frac{\mathrm{d} Q}{\mathrm{~d} \lambda}}\right)^{2} \mathrm{~d} \lambda
$$

where $P$ and $Q$ denote two probability distributions, respectively.

For two discrete histograms $H_{i}$ and $H_{j}$, the Hellinger distance is computed as the contrast between $R_{i}$ and $R_{j}$ :

$$
H\left(H\left(R_{i}\right), H\left(R_{j}\right)\right)=\frac{1}{\sqrt{2}} \sqrt{\sum_{k=1}^{b}\left(\sqrt{h_{i k}}-\sqrt{h_{j k}}\right)^{2}}
$$


(4) Intervening Contour Distance: The intervening contour distance [44] employs the edge probability map to measure the difference between two superpixels, which can provide distinction when salient object and background share similar color features. It is defined as:

$$
I\left(R_{i}, R_{j}\right)=\max _{l \in \overline{R_{i} R_{j}}} E(l)
$$

where $\overline{R_{i} R_{j}}$ is the straight line connecting the pixels of superpixels $R_{i}$ and $R_{j}, l$ runs over every line segment between the two superpixels, and $E(l)$ is the corresponding edge probability map.

Table 1

Features and corresponding distance metrics we used to construct multiple graphs.

\begin{tabular}{cc}
\hline Feature description & Distance metric \\
\hline Average CIELab color & $L_{2}$ \\
Edge probability map & Intervening contour \\
Global contrast of color & $L_{2}$ \\
Local contrast of color & $L_{2}$ \\
Gaussian distribution of color & $W_{2}$ \\
Color histogram & Hellinger \\
\hline
\end{tabular}

\subsection{Multi-graph construction}

As described in section 1, we construct multiple graphs by using multiple distance metrics to measure multiple features. We first construct an undirected graph $G=\langle V, E\rangle$ as shown in Fig. 4(b) to reveal the connection relationships of superpixels, where the vertices $V$ are superpixels and the edges $E$ are the relevance between $R_{i}$ and $R_{j}\left(R_{i}, R_{j} \in R\right)$. The edge weights reveal the similarity between superpixels which are defined by an affine matrix $W \in \mathrm{R}^{N \times N}$. Table 1 lists the features and corresponding distance metrics we used, and we can easily generate multiple graphs $W_{k}$ $(k=1, \ldots, 6)$ as follows:

$$
w_{k, i j}= \begin{cases}\exp \left(-d\left(R_{k, i}, R_{k, j}\right) / \delta_{k}\right), & \text { if } R_{k, i} \text { and } R_{k, j} \text { are connected } \\ 0, & \text { otherwise }\end{cases}
$$

where $d\left(R_{k, i}, R_{k, j}\right)$ denotes the distance of the $k^{\text {th }}$ feature between the superpixels $R_{i}$ and $R_{j}$, and $\delta_{k}$ is a constant to control the strength of weight. Compared with the conventional methods [11], [15] which use a fixed control parameter, One of the contributions of our algorithm is to set different values of $\delta_{k}$ for different graphs to reflect feature characteristics. Here, these parameters are decided by 10 -fold cross-validation method. In our work, we finally get six graphs $W_{1}, W_{2}, W_{3}, W_{4}, W_{5}$, and $W_{6}$. Clearly, all the graphs are sparse matrix because of the constraints on edges.

Like [11], the nodes are not only connected to the direct neighboring nodes, but also connected to the direct neighbors of those neighbors. It is obvious that the locally connected graphs might lose global relationships with all the other superpixels. In our work, global and local contrast graphs are introduced to aggregate complementary appearance contrast measures, which make locally connected graphs more effective to consider both of global and local consistency simultaneously. In addition, connecting all the boundary nodes is proved to be effective to improve performance, because this close-loop structure considers geodesic distance [45]. In summary, our multiple graphs are represented as follows:

$$
W_{k}= \begin{cases}w_{k, i j}, & \text { if } j \in n(i) \text { or } i, j \in B \\ 0, & \text { otherwise }\end{cases}
$$

where $n(i)$ denotes all nodes are connected to the node $i$ and $B$ is the set of boundary nodes. The diagonal entries of $W_{k}$ are set to zeros to prevent self-loops in the graph. 


\subsection{Seed extraction}

Different from most previous methods that mainly concentrate on image object or background, we take both background and foreground into consideration. Foreground seeds and background seeds are collected by eye fixations and boundary information, respectively.

\subsubsection{Foreground seeds}

Previous works generate foreground seeds by boundary priors or rare shapes, which is not robust for saliency detection in complex environments. Unlike theirs, based on the agreement between fixation and saliency judgments that the most salient object in a scene is the one that attracts the majority of fixations [46], we take regions where eye fixations follow as foreground seeds to improve the accuracy of hitting salient objects. For totally automatic saliency detection, eye fixations need to be automatically predicted by computational models. It is worth noting that any fixation model that outputs fixation map can be employed. Here, we adopt the BMS [47] to provide the fixation saliency map because it is one of the best models for simulating human fixations validated by [13].

After getting the fixation prediction map, we first normalize it to $(0,1)$, and then threshold it at $\beta$ (here $\beta=0.7$ ) to obtain the binary map. Suppose the binary map consists of two parts: the candidate foreground (salient regions, may contain some background regions) and the pure background (non-salient regions). We utilize a shift convex hull strategy to approximately acquire foreground seeds from the binary map. Specifically, we construct a convex hull $C$ (the green polygon shown in Fig. 4(c)) based on the candidate foreground of the binary map. Then in order to maximize the probability of covering salient object, we select all unique superpixels that spatially neighbor with $C$ as foreground seeds (the blue polygon shown in Fig. 4(c)). Since the image boundary is most likely to be background [45], we discard those superpixels that touch the image boundary.

\subsubsection{Background seeds}

Since the superpixels that touch the image boundary are highly likely to be part of background, we choose the boundary superpixels as background seeds (the yellow polygon shown in Fig. 4(c)). In our work, we use the $S C$ approach proposed by [11] to generate saliency maps. In the $S C$, the superpixels on each boundary serve as background seeds to evaluate the saliency, which lead to four saliency maps. Then the four saliency maps are integrated with the multiplication operation to produce the background saliency map. In addition, when foreground seeds contain boundary nodes after the above-mentioned operation, we suppose that the nodes belong to foreground seeds. When the background seeds have high contrast which may not belong to the background seeds, we discard those seeds from the background seeds.

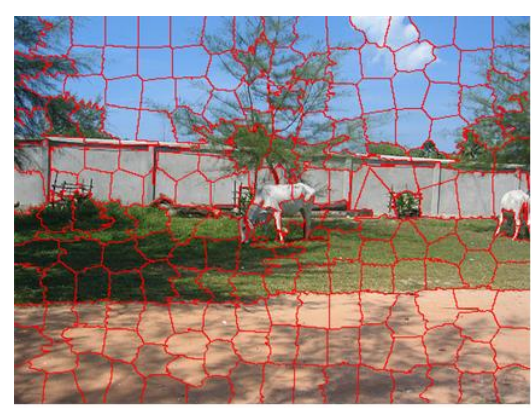

(a)

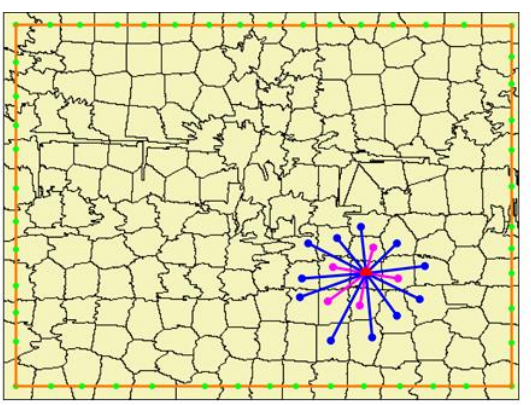

(b)

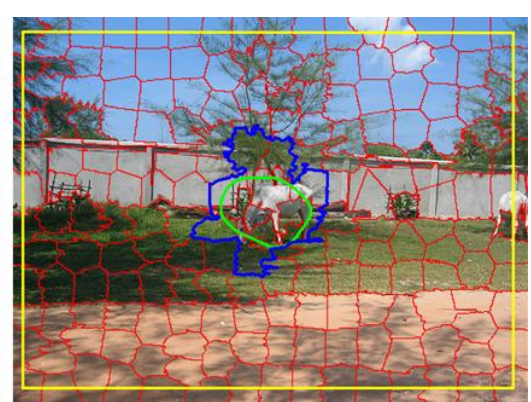

(c)

Fig. 4. Illustration of superpixel extraction, the corresponding connection relationship of superpixels, and seed extraction. From left to right: (a) the superpixel map. (b) the connection relationships of superpixels. The magenta lines represent the direct neighboring superpixels, the blue lines represent the neighbors of those neighbors, and the orange lines indicate that all the boundary superpixels are connected to each other. (c) the blue and green polygons denote foreground seeds and convex hull $C$, respectively. The superpixels intersecting with yellow polygon are the background seeds. 


\subsection{Saliency estimation with single graph}

Graph based semi-supervised learning methods are conducted on a graph, where the vertices are labeled and unlabeled superpixels and the edges reflect the similarities between superpixel pairs. We consider the method proposed by [48] to perform saliency estimation with both of saliency and background seeds. For single graph $W_{k}(k=1, \ldots, 6)$, the degree matrix $D$ of $W_{k}$ is:

$$
\left\{\begin{array}{l}
D=\operatorname{diag}\left\{d_{11}, \ldots, d_{N N}\right\} \\
d_{i i}=\sum_{j=1}^{N} w_{k, i j}, \quad i=1,2, \ldots, N
\end{array}\right.
$$

Given foreground seeds and background seeds, we need to let every seed iteratively spread its saliency information to its neighbors until a global stable state is achieved. Therefore, a semi-supervised learning method could be constructed:

$$
F(t+1)=\alpha S F(t)+(1-\alpha) Y
$$

where $\alpha$ is a trade-off between the neighbors of superpixel and initial information in $(0,1), S=D^{-1 / 2} W_{k} D^{-1 / 2}$ is the symmetric normalized Laplacian matrix, $F=\left[f_{1}, f_{2}, \ldots, f_{n}\right]^{T}$ indicates the calculated saliency values at time $t$, and $Y=\left[y_{1}, y_{2}, \ldots, y_{n}\right]^{T}$ denotes an indication vector, in which $y_{i}=1$ if $R_{i}$ is a foreground seed or background seed, and $y_{i}=0$ otherwise. A regularization framework is developed to rewrite Eq. (17) by solving the following optimization problem:

$$
F^{*}=\arg \min _{f} \frac{1}{2}\left(\sum_{i, j=1}^{N} w_{k, i j}\left\|\frac{f_{i}}{\sqrt{d_{i i}}}-\frac{f_{j}}{\sqrt{d_{j j}}}\right\|^{2}+\frac{1-\alpha}{\alpha} \sum_{i=1}^{N}\left\|f_{i}-y_{i}\right\|^{2}\right)
$$

Let $L=\mathrm{I}-S$ and $\mu=1 / \alpha-1$. By setting the derivative of $F$ to be zero, and the saliency estimation is conducted with the saliency and background seeds as:

$$
S_{f}=\left(\mathrm{I}+\frac{1}{\mu} L\right)^{-1} Y_{f} \quad S_{b}=\left(\mathrm{I}+\frac{1}{\mu} L\right)^{-1} Y_{b}
$$

where $Y_{f}$ and $Y_{b}$ denote the foreground seeds and background seeds, respectively. Finally, we normalize both foreground and background saliency maps to the range of $(0,1)$ and obtain the combined saliency map by integrating the two kinds of saliency maps. In practice, we find that the background saliency map $S_{b}$ has more discriminative power. Therefore, we adopt an exponential function in order to emphasize $S_{b}$ which is defined as:

$$
S_{c}=S_{f} * \exp \left(k_{s} S_{b}\right)
$$

where $*$ denotes the superpixel-wise multiplication of two vectors. In all our experiments, we set $k_{s}=15$ as the scaling factor. Finally, the combined superpixel saliency map $S_{c}$ is normalized to $(0,1)$.

More frequently, it is solved by an iterative process. The main iterative steps of the single-graph based saliency estimation are summarized in Algorithm 1.

Fig. 5(b)-(g) show the saliency estimation result with six graphs. For the first example image, which has a dog object with heterogeneous regions and a clean background, all the saliency maps succeed to detect the salient object. However, when a scene contains a salient object with cluttered background as shown in the second example image, each saliency map alone fails to highlight the entire bird out. In certain cases, an edge feature provides better delineation between salient object and background than other features. For the last example image, all the other saliency maps fail to plot the entire egret as the weak color difference between the egret and background, but the edge feature based saliency map well highlights the entire egret. It is obvious that the single-graph based saliency measurement is not enough to detect salient objects. The best way to handle the shortcomings is that using the complementarity of multiple features and performing multi-graph based saliency estimation deal with various scenes. 


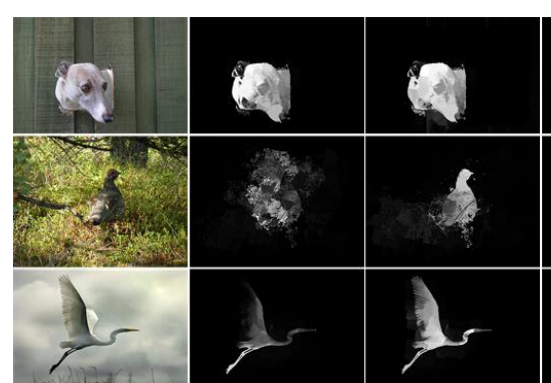

(a)

(b)

(c)

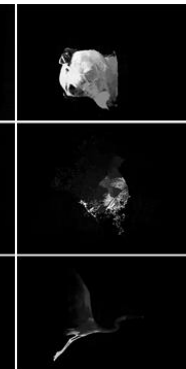

(d)

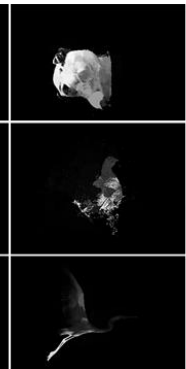

(e)

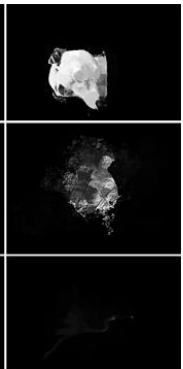

(f)

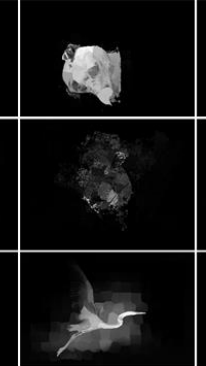

(g)

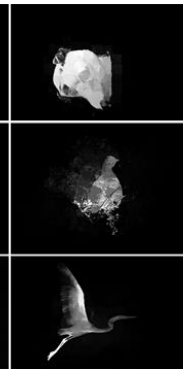

(h)

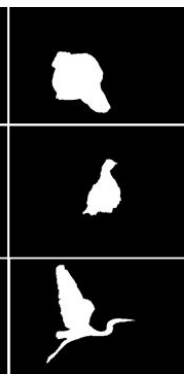

(i)

Fig. 5. Illustration of saliency estimation with single graph and multiple graphs. (a) original image; (b) color graph based saliency map; (c) edge probability graph based saliency map; (d) global contrast graph based saliency map; (e) local contrast graph based saliency map; (f) normal distribution graph based saliency map; (g) color histogram graph based saliency map; (h) multi-graph based saliency map (i) the ground truth.

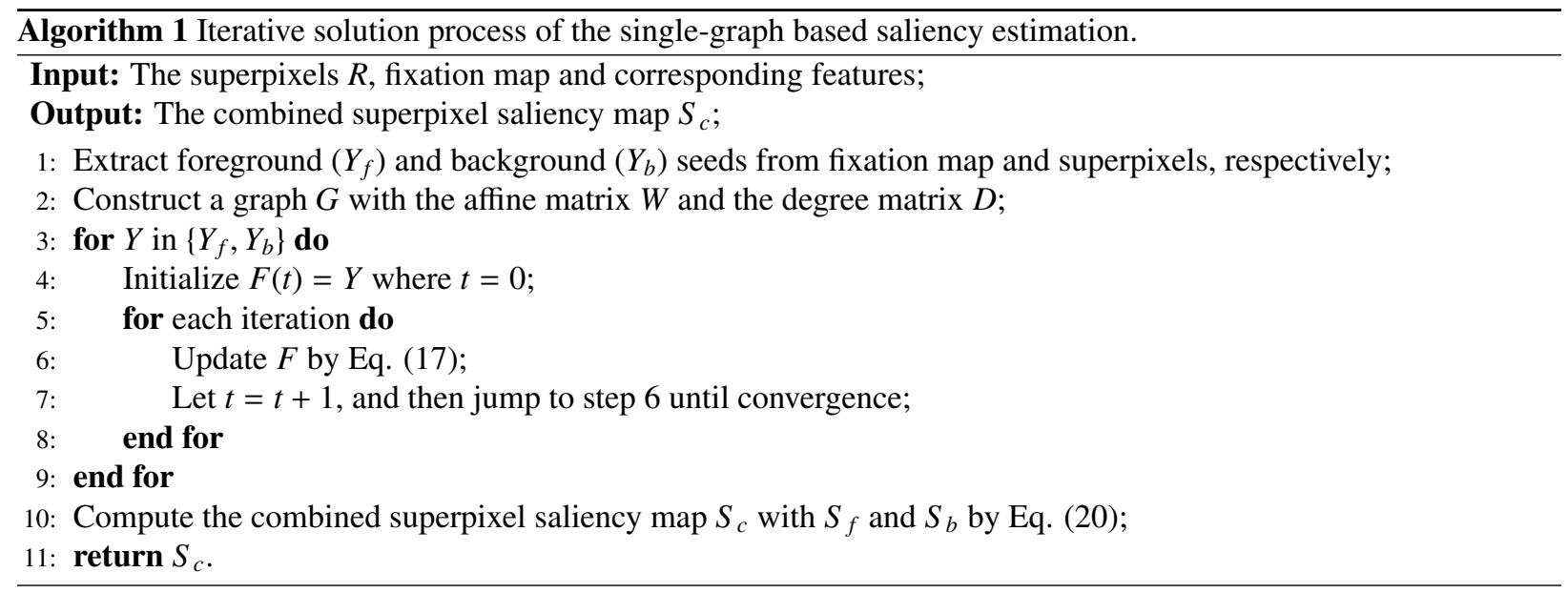

\subsection{Saliency estimation with optimized multiple graphs}

(1) Regularization framework: As stated previously, the single-graph based learning is insufficient to perform saliency estimation. In section 3.3, we have obtained $K(K=6)$ graphs. Now our goal is to utilize the complementarity of those graphs to improve saliency estimation performance. A multi-graph based learning algorithm has been proposed by [49]. It is able to integrate multiple complementary graphs into a regularization framework. So we modify the regularization framework Eq. (18) to satisfy multiple graphs as follows:

$$
F^{*}=\arg \min _{f} \frac{1}{2}\left(\sum_{k=1}^{K} \sum_{i, j=1}^{N} \varphi_{k}\left(w_{k, i j}\left\|\frac{f_{i}}{\sqrt{d_{k, i i}}}-\frac{f_{j}}{\sqrt{d_{k, j j}}}\right\|^{2}+\frac{1-\alpha}{\alpha} \sum_{i=1}^{N}\left\|f_{i}-y_{i}\right\|^{2}\right)\right)
$$

where $\varphi=\left[\varphi_{1}, \varphi_{2}, \ldots, \varphi_{K}\right]$ is the weight vector which reflects the utilities of different graphs. We can easily derive the closed-form solution from Eq. (21) as:

$$
F=\left(\mathrm{I}+\frac{1}{\mu} \sum_{k=1}^{K} \varphi_{k} L_{k}\right)^{-1} Y
$$

where $L_{k}=\mathrm{I}-D_{k}^{-1 / 2} W_{k} D_{k}^{-1 / 2}$ is the normalized graph Laplacian obtained from the graph $W_{k}$.

(2) Optimized multi-graph fusion: Since the discriminative abilities may vary intensively among different features, it is crucial to vary $\varphi_{k}$ according to their discriminative abilities. In order to automatically to achieve this, we also regard $\varphi_{k}$ as variables in Eq. (21) and make a relaxation by changing $\varphi_{k}$ to $\varphi_{k}^{r}(r>1)$. Note that $\sum_{k=1}^{K} \varphi_{k}^{r}$ achieves its 
minimum when $\varphi_{k}=1 / K$ with the constraint $\sum_{k=1}^{K} \varphi_{k}=1$. Then the joint optimization of $F$ and $\varphi$ is solved by using the alternative optimization technique, which iteratively optimizes $F$ with fixed $\varphi$ and optimizes $\varphi$ with fixed $F$ until convergence [9]. The solution of $F$ and $\varphi$ can be obtained as:

$$
\begin{gathered}
\varphi_{k}=\frac{\left(\frac{1}{f^{T} L_{k} f+\mu\|f-Y\|^{2}}\right)^{1 /(r-1)}}{\sum_{k=1}^{K}\left(\frac{1}{f^{T} L_{k} f+\mu\|f-Y\|^{2}}\right)^{1 /(r-1)}} \\
F=\left(\mathrm{I}+\frac{1}{\mu} \frac{\sum_{k=1}^{K} \varphi_{k}^{r} L_{k}}{\sum_{k=1}^{K} \varphi_{k}^{r}}\right)^{-1} Y
\end{gathered}
$$

From the above derivation, the iterative process of the optimized multi-graph based saliency estimation is illustrated in Algorithm 2. By integrating the six graphs which generate the saliency maps in Fig. 5(b)-(g), the saliency map shown in Fig. 5(h) can highlight salient object regions either in clutter scene or in similar color scene between foreground and background.

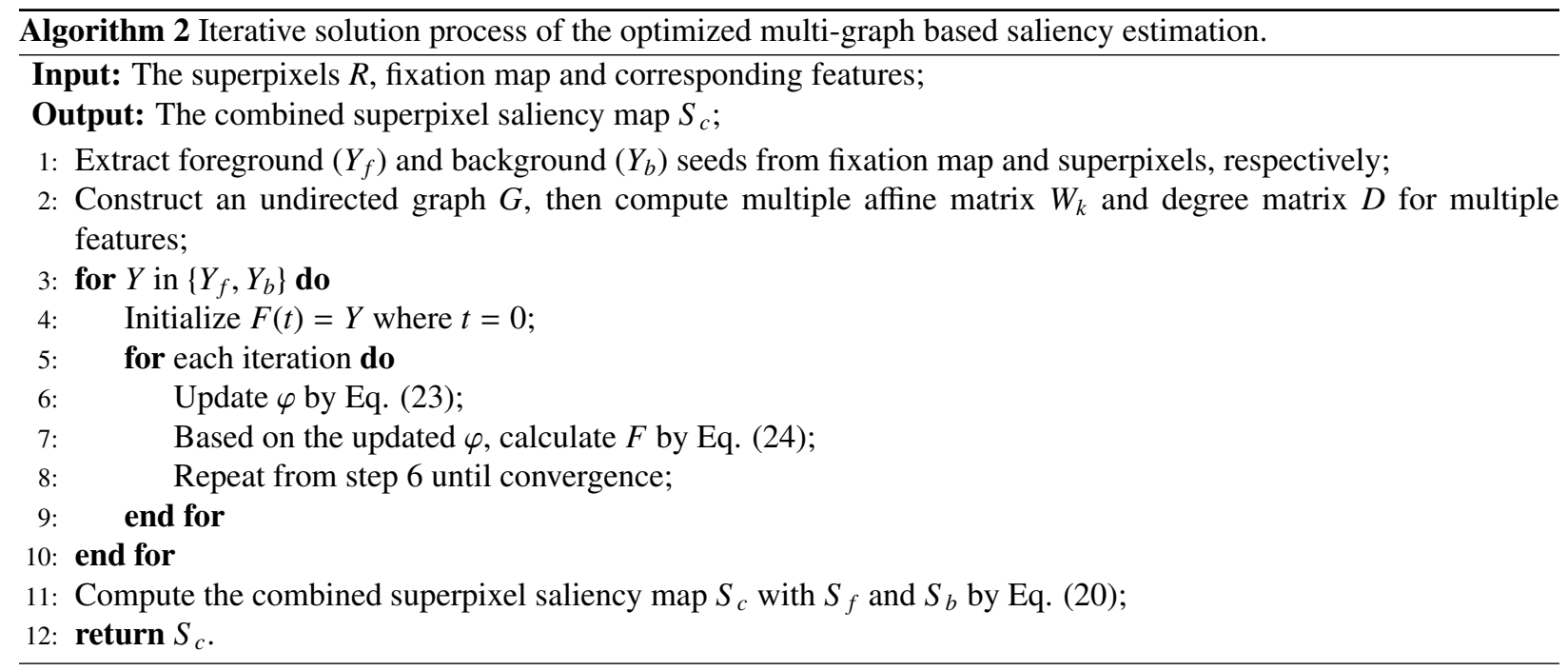

\subsection{Pixel-level saliency map generation}

Finally, we need to assign the combined superpixel saliency value to each image pixel. Similar to [50] and [51], the saliency value of each pixel is defined as the weighted sum of superpixel where the pixel is and adjacent superpixels:

$$
S(p)=\sum_{k=1}^{M} \exp \left(-k_{1}\left\|c_{p}-c_{k}\right\|-k_{2}\left\|z_{p}-z_{k}\right\|\right) S\left(R_{k}\right)
$$

where $c_{p}$ and $z_{p}$ are the color and coordinate values of the $p^{\text {th }}$ pixel, $c_{k}$ and $z_{k}$ are the color and coordinate values of a superpixel, $M$ denotes the number of direct neighbors of the superpixel where the $p^{\text {th }}$ pixel is, and $S\left(R_{k}\right)$ is the superpixel-level saliency value from Algorithm 1 or Algorithm 2. Here $k_{1}$ and $k_{2}$ are parameters to control the effect of color and position. We find $k_{1}=0.1$ and $k_{2}=0.01$ to work well in practice. 


\section{Experimental results and analysis}

In this section, we exhaustively compare our optimized multi-graph based saliency detection method with sixteen state-of-the-art saliency detection methods on five widely used datasets.

\subsection{Evaluation datasets}

The proposed method is evaluated on five publicly available datasets: MSRA-B [9], DUT-OMRON [11], ECSSD [52], PASCAL-S [32], and Judd-A [46]. MSRA-B and DUT-OMRON are used to compare methods on a large scale; ECSSD contains semantically meaningful but structurally complex images; PASCAL-S and Judd-A usually contains multiple objects with high-clutter backgrounds; and SED2 is included to evaluate whether salient models can perform well on images containing multiple objects with clean background.

More specifically, MSRA-B contains 5000 images with manually labeled pixel-wise ground truth provided by [41]. Similar to MSRA-B, the 5168 images of DUT-OMRON are also created from more than 140000 natural images, each of which has one or more salient objects and relatively complex backgrounds. As an extension of the Complex Scene Saliency Dataset (CSSD), ECSSD includes 1000 semantically meaningful but structurally complex images which are obtained from the BSD dataset, PASCAL VOC and the Internet. Judd dataset [53] is the most popular dataset which contains 1003 images for benchmarking fixation prediction models. Judd-A [46] selects 900 images from Judd and annotates the most salient objects so that it can be used for benchmarking salient object detection models. PASCAL-S is built on the validation set of the PASCAL VOC 2010 segmentation challenge. It contains 850 natural images which are labeled with pixel-wise ground truth.

Fig. 6 lists the statistical information of five datasets to analysis the complexity of scenes on five datasets. Fig. 6(a) shows the distributions of the number of superpixels on each dataset. As we can see, the entire scenes in PASCAL$\mathrm{S}$ and Judd-A datasets on average contain more superpixels than that of other datasets, which illustrates these two datasets are more complex. Furthermore, Judd-A dataset is more complex and cluttered than other datasets.

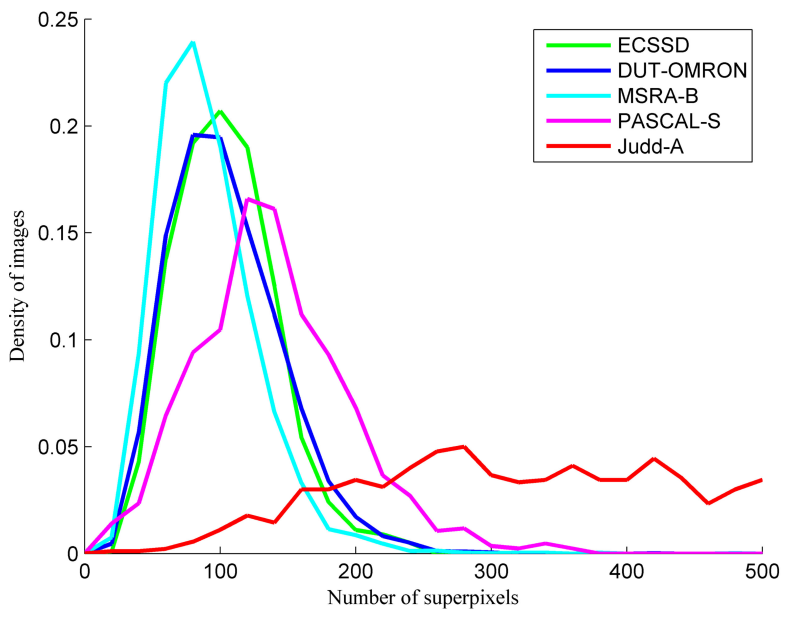

(a)

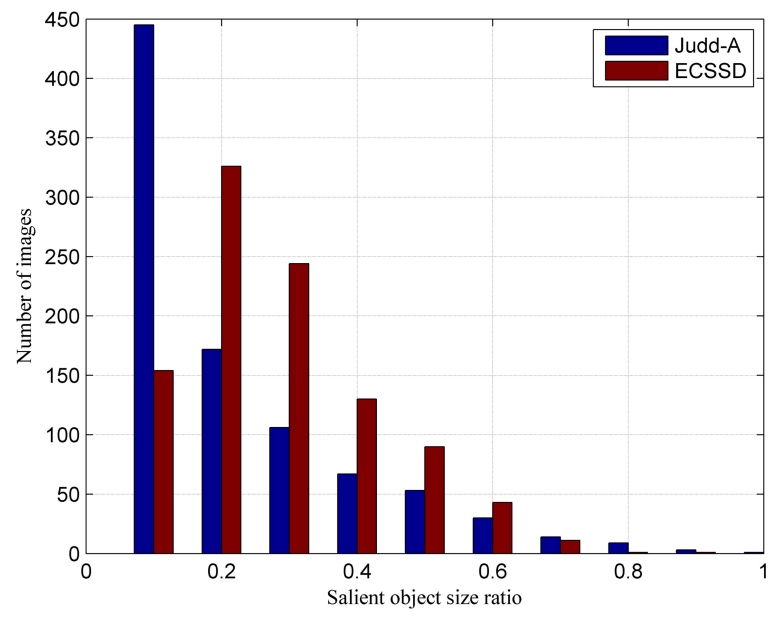

(b)

Fig. 6. The statistical information of five datasets. (a): distributions of the number of superpixels for the entire scene across five datasets. (b): distribution of salient object size ratio across ECSSD and Judd-A datasets.

\subsection{Evaluation metrics}

In the experiments, we use three widely adopted metrics for qualitative and quantitative comparison:

(1) Precision-Recall (PR) Curve: The PR curve indicates the capacity of models to retrieve salient objects. For a saliency map, we obtain a binary map $B$ by segmenting it with a fixed threshold $T \in(0,255)$, then precision and recall are calculated with the ground truth $G$ as follows: 


$$
\text { Precision }=\frac{B \cap G}{B}, \text { Recall }=\frac{B \cap G}{G}
$$

Varying the threshold $\mathrm{T}$ within $(0,255)$, we get 256 pairs of precision and recall. Finally, we average both precision and recall of all images in the dataset to plot the PR curve.

(2) F-measure: The F-measure is computed to comprehensively evaluate the performance of models defined as:

$$
F_{\gamma}=\frac{(1+\gamma) \text { Precision } \times \text { Recall }}{\gamma \times \text { Precision }+ \text { Recall }}
$$

where $\gamma$ is set as 0.3 as suggested by [54] to emphasize precision.

(3) Receiver Operating Characteristics (ROC) Curve: Similar to PR curve, the ROC curve is the plot of false positive rate $(F P R)$ versus true positive rate (TPR) with fixed thresholds, which is defined as:

$$
T P R=\frac{B \cap G}{G}, F P R=\frac{B \cap \bar{G}}{\bar{G}}
$$

where $\bar{G}$ denotes the complement of the ground truth $G$. The ROC curve is plotted by varying the fixed threshold from 0 to 255 .

\subsection{Performance evaluation}

We quantitatively and qualitatively compare the proposed method with sixteen state-of-the-art saliency detection methods, including IT [8], FT [54], RC [14], SF [50], MC [15], DSR [16], GMR [11], HC [14], HS [52], RBD [17], ST [18], GC [55], MB+ [56], MST [57], LPS [51], and HDCT [24]. The implementation of each method is provided by authors to generate saliency maps.

Fig. 7 shows the PR curves, ROC curves and F-measure of all the compared methods on the five benchmark datasets, and we take the maximum $F_{\gamma}$ to be the F-measure. Table 2 shows the F-measure with all the compared methods on the five datasets. As seen in Fig. 7 and Table 2, our proposed method achieves comparable or better performance against the best methods on all the five datasets. Specifically, for the MSRA-B, DUT-OMRON and ECSSD datasets, the F-measure is $2.3 \%, 0.6 \%$ and $1.2 \%$ less than the best models, respectively. Though our method is a little worse than the best methods on the three datasets, it has proved that higher F-measure is achievable using the fixation model LDS [58] (in section 4.4). For simple scene, each feature we used is enough to represent the salient object and the complementarity of these features is weak, it is not suitable for showing the strengths of our method that exploit multiple features to cope with variant clutter backgrounds. Moving from simple datasets (e.g., MSRA-B) to more complex datasets (e.g., PASCAL-S and Judd-A), we can observe a dramatic degradation in the performance of all methods. The average drop from MSRK-B to Judd-A dataset is $45.8 \%$ over the F-measure (for all the compared methods except ours). Now, one can clearly see that our method consistently outperforms all the compared methods on PASCAL-S and Judd-A. In particular, the salient objects of Judd-A contain diverse sizes and complex structure information with various backgrounds. Despite that, our method achieves an improvement of $4.8 \%$ in comparison to the second best method MB+. This is consistent with our motivation that utilizes the complementarity of multiple graphs to deal with different scenes and detect salient objects, and our method is more robust for various scenes with simple and complex background against other saliency methods.

Fig. 12 shows visual comparisons of our method over several challenging scenes against the other state-of-the-art methods. As can be seen, our method effectively suppresses background clutter and detects salient objects. Especially, our method can handle the challenging cases that the state-of-the-art methods fail. Such as the 10th, 11th, and 12th row in Fig. 12, our method succeeds in detecting salient objects and suppressing background objects at the same time. Benefiting from the edge probability cue, our algorithm also can detect salient objects in scenes, which have similar color between foreground and background (e.g., the 9th row in Fig. 12). In addition, our approach tends to highlight the salient object with different size because eye fixations can hit the salient object and detect it (e.g., the 3rd and 4th rows in Fig. 12). Apparently, the proposed method owns better robustness either in clean backgrounds or high-cluttered scenes. 

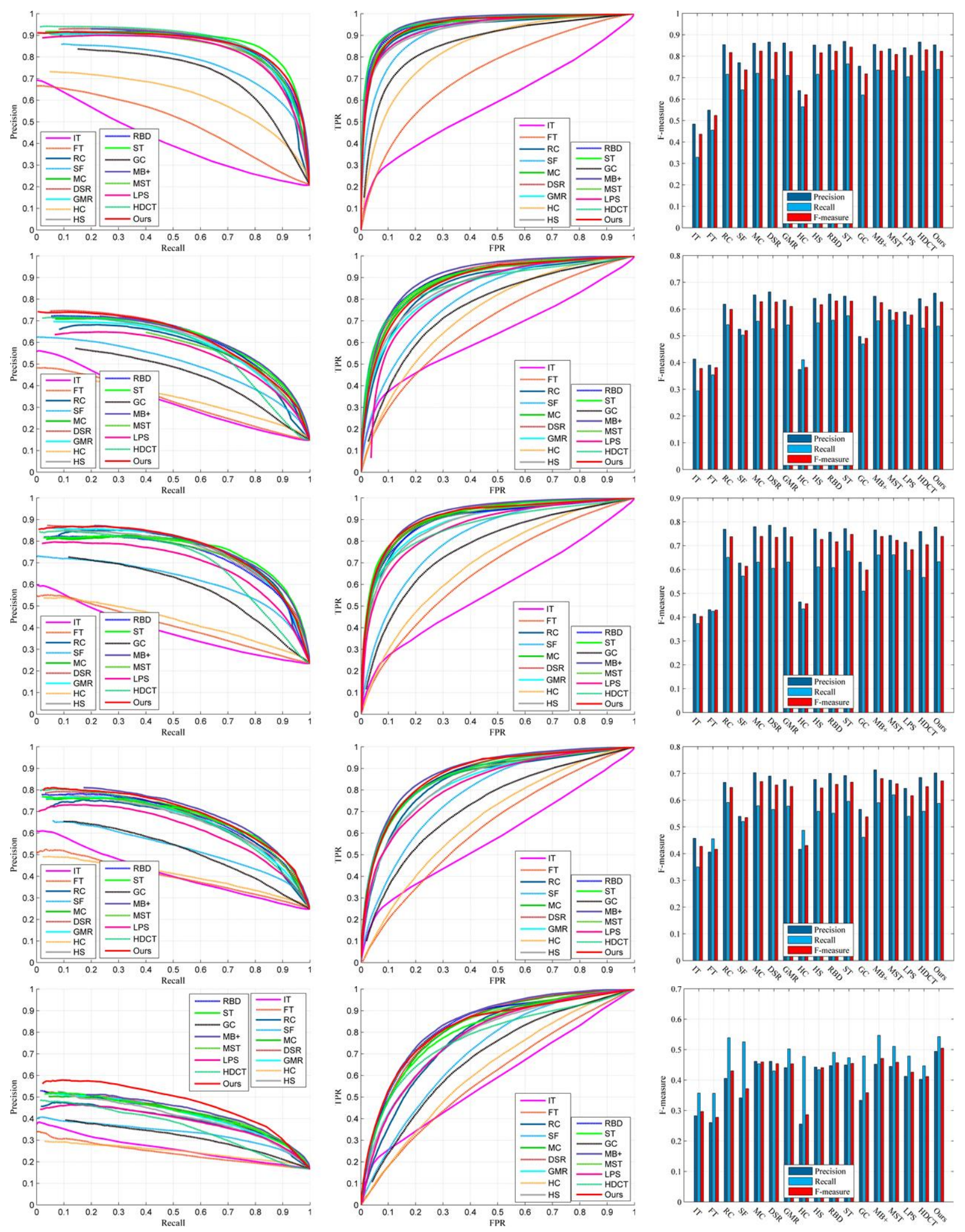

Fig. 7. Quantitative comparison of our methods with state-of-the-art methods on five widely used benchmark datasets. From top to down: MSRA-B, DUT-OMRON, ECSSD, PASCAL-S, and Judd-A datasets. From left to right: (a): the PR curves. (b): the ROC curves. (c): the F-measure. 
Table 2

Comparison of F-measure with state-of-the-art algorithms on five widely used benchmark datasets. The three best results are highlighted in red, green, and blue bold fonts, respectively.

\begin{tabular}{ccccccccccccccccccc}
\hline Dataset & IT & FT & RC & SF & MC & DSR & GMR & HC & HS & RBD & ST & GC & MB+ & MST & LPS & HDCT & Ours \\
\hline MSRA-B & 0.436 & 0.524 & 0.817 & 0.736 & 0.824 & 0.818 & 0.821 & 0.620 & 0.816 & 0.823 & 0.842 & 0.717 & 0.824 & 0.808 & 0.804 & 0.830 & 0.823 \\
DUT-OMRON & 0.378 & 0.381 & 0.599 & 0.519 & 0.627 & 0.626 & 0.610 & 0.382 & 0.616 & 0.631 & 0.630 & 0.491 & 0.624 & 0.588 & 0.578 & 0.609 & 0.626 \\
ECSSD & 0.403 & 0.430 & 0.738 & 0.614 & 0.739 & 0.735 & 0.738 & 0.457 & 0.727 & 0.716 & 0.748 & 0.598 & 0.739 & 0.723 & 0.683 & 0.704 & 0.739 \\
PASCAL-S & 0.427 & 0.416 & 0.647 & 0.535 & 0.669 & 0.657 & 0.651 & 0.431 & 0.645 & 0.659 & 0.667 & 0.537 & 0.681 & 0.661 & 0.617 & 0.651 & 0.672 \\
Judd-A & 0.297 & 0.278 & 0.430 & 0.372 & 0.460 & 0.454 & 0.454 & 0.286 & 0.441 & 0.457 & 0.455 & 0.359 & 0.471 & 0.459 & 0.426 & 0.412 & 0.505 \\
\hline
\end{tabular}

\subsection{Components analysis}

We further analyze the effectiveness of different components: the influence of fixation method, the effect of multiple graphs, the effectiveness of optimized fusion, and the parameter $r$ of saliency estimation.

(1) Analysis of fixation method: The fixations in the paper are used to indicate the salient objects and then served as seeds to perform the multi-graph based saliency estimation. Therefore, it is important for fixation prediction model to accurately locate salient objects for our method. To study the influence of fixation prediction model, we calculate the three metrics to compare the fixation model BMS we used and some latest models LDS [58] and SSD [59] on PASCAL-S dataset. We observe the F-measure of BMS is higher than that of SSD but lower than that of LDS. The reason is that the fixations predicted by SSD are more scattered, which increases the failure of hitting salient objects. The opposite holds over the LDS.
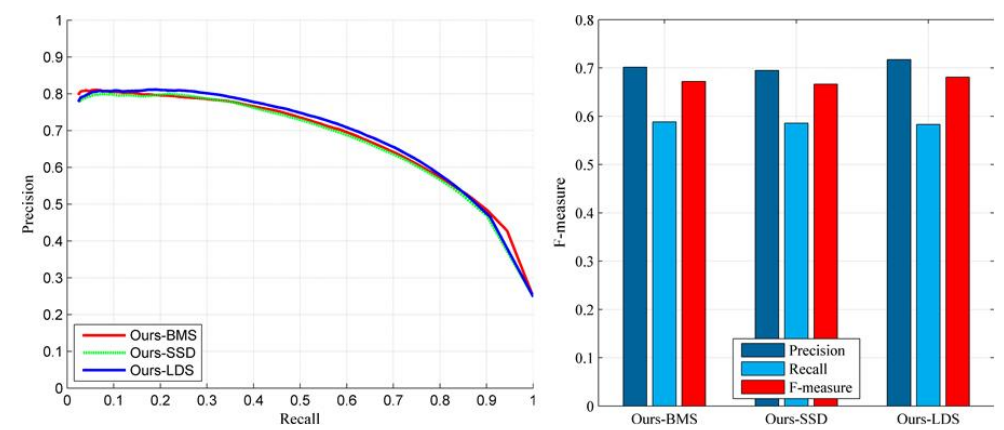

Fig. 8. Comparison of different fixation prediction methods on PASCAL-S dataset.

(2) Analysis of multiple graphs: To investigate the effectiveness of the multi-graph based saliency detection, we compare the PR curve and F-measure of single-graph based saliency with multi-graph based saliency on PASCAL$\mathrm{S}$ dataset. As shown in Fig. 9, all the evaluation metrics of multi-graph based saliency detection grow by a large margin. Specifically, compared with edge probability based method achieving the worst performance, the F-measure of multi-graph based saliency increases by $11.7 \%$, and the Precision and Recall of multi-graph based saliency increase by $15.9 \%$ and $0.1 \%$, respectively. Even if compared with the normal distribution based method which is the best in the six single-graph based methods, the F-measure, Precision and Recall of multi-graph based method also increase by $6.6 \%, 8.1 \%$ and $2.5 \%$, respectively. Considering that the PASCAL-S dataset is more complex than other datasets except Judd-A, the improvement is enough to illustrate the effectiveness of our optimized multi-graph based saliency method.

(3) Analysis of optimized fusion: In our paper, we assign different weights for multiple graphs to reflect there discriminative abilities to optimize the multi-graph fusion. In order to illustrate the effectiveness of the optimized multi-graph fusion, we compare the optimized fusion of multi-graph with the direct fusion without weights of multigraph. As shown in Fig. 10, all the evaluation metrics of the optimized multi-graph fusion grow by a large margin, which illustrates the effectiveness of the varying weights for different features. Meanwhile, the F-measure increase by $7.7 \%$ on Judd-A but $3.3 \%$ on MSRA-B, which explains the optimized fusion can take better advantage of their discriminative abilities on complex scenes compared with direct fusion. 

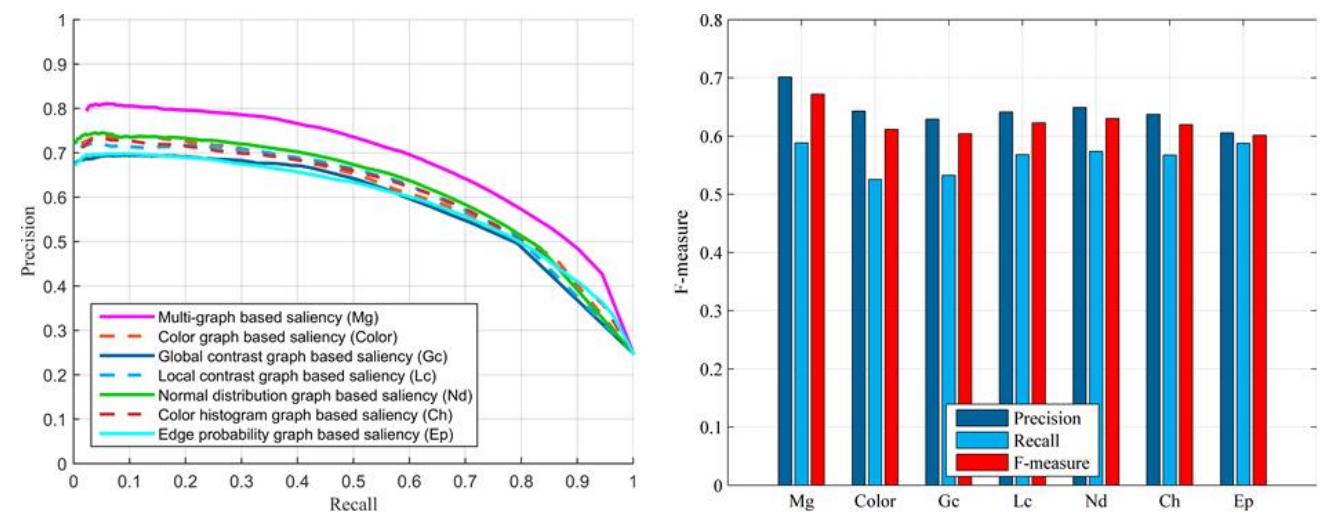

Fig. 9. Comparisons of multi-graph based saliency and single-graph based saliency on PASCAL-S dataset. (a): the PR curve of multi-graph based and six single-graph based saliency methods. (b): the F-measure of multi-graph based and six single-graph based saliency methods.

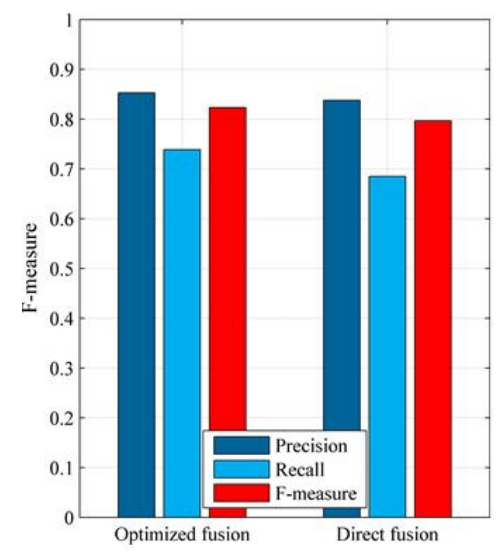

(a) MSRA-B

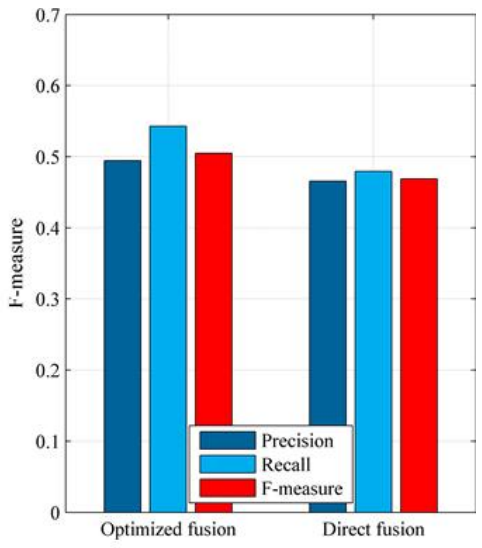

(b) Judd-A

Fig. 10. Comparison of the optimized fusion and direct fusion on MSRA-B and Judd-A dataset.

(4) Analysis of parameter $r$ : In order to study the relationship between $r$ and performance in our method, we vary the parameter $r$ in Eq. (23) and Eq. (24), and calculate the corresponding F-measure on ECSSD and PASCAL-S datasets. As shown in Fig. 11, the ECSSD and PASCAL-S datasets exhibit the similar curves. When $r$ increases from 1 to $\infty$, the F-measure firstly increases and then reaches steady. This is because the complementary nature of multiple graphs has not been sufficiently explored when $r$ is near 1 and the graphs are well fused when $r$ is greater than 5. From the two figures we can see that the performance improvement is very limited as the parameter $r$ increases from 10 . Hence in practice we set $r=10$ to conduct all the experiments.

\subsection{Running time}

To further demonstrate the efficiency of our algorithm, we compare the average computational time for the proposed method with the other state-of-the-art methods mentioned above. The proposed method is implemented in MATLAB and the experiments are carried out on an HP workstation with an Intel i7-3720QM 2.6GHz CPU and 8GB RAM. Table 3 shows a comparison of the average running time on ECSSD dataset, whose images have an average resolution of $311 \times 375$. The top three methods are all implemented using $\mathrm{C}++$, and our method is better than these methods in terms of performance (see Fig. 7). Meanwhile, considering that our method is implemented by using MATLAB with unoptimized code, the computational complexity of the proposed method is comparable to that of other methods. 

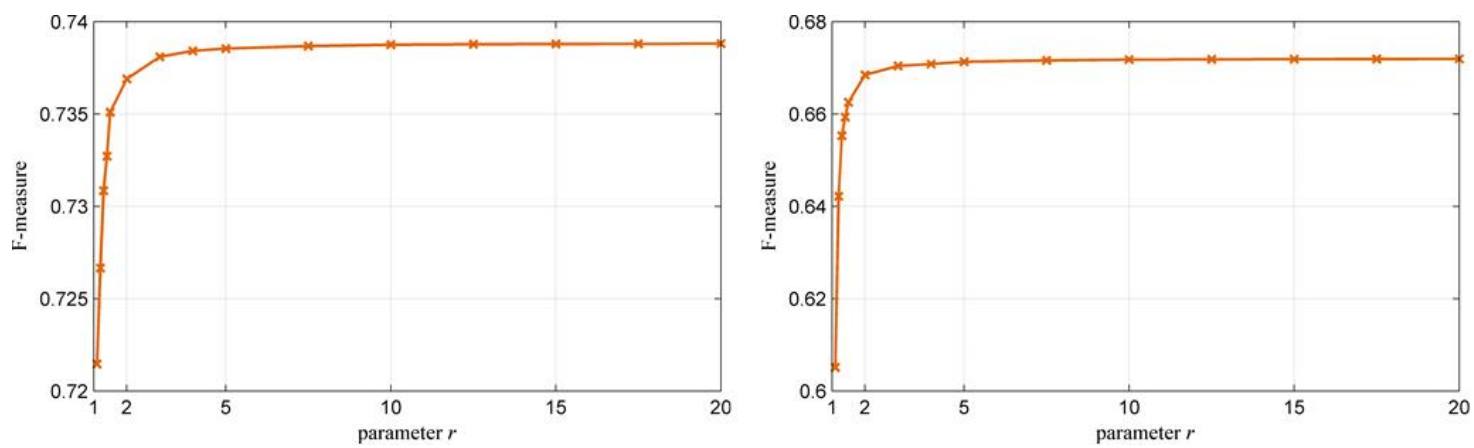

Fig. 11. F-measure with respect to $r$ on ECSSD and PASCAL-S dataset. The two datasets exhibit the similar curves.

Table 3

Comparison of the average running time (seconds per image) on the ECSSD dataset.

\begin{tabular}{ccccccccccccccccc}
\hline Method & Ours & IT & FT & RC & SF & MC & DSR & GMR & HC & HS & RBD & GC & MB+ & MST & LPS & HDCT \\
\hline Time(s) & 1.847 & 0.183 & 0.089 & 0.087 & 0.131 & 0.184 & 3.327 & 0.653 & 0.012 & 0.384 & 0.254 & 0.028 & 0.019 & 0.256 & 1.950 & 5.477 \\
Code & Matlab & Matlab & Matlab & C++ & C++ & Matlab & Matlab & Matlab & C++ & C++ & Matlab & C++ + C++ & C++ & Matlab & Matlab \\
\hline
\end{tabular}

\subsection{Limitations}

Although our method effectively suppresses background clutter and detects salient objects, it still has some limitations. For example, if similar colors appear in both the foreground and the background (e.g., the 9th row in Fig. 12) or the color contrast between them is low (e.g., the 2nd row in Fig. 5), our method fails to completely suppress the background and generates scattered small regions around salient objects. The reason is that all features we used except the edge probability are related to color, and when the color features are invalid for those scenes, our method is unsatisfying to segment salient objects out. However, this shortcoming can be addressed by introducing more priors that are uncorrelated with color such as the shape of a superpixel [32] and concavity [60]. In addition, as our approach uses fixation prediction to indicate the most salient object in scenes, it has problems when scenes contain multiple objects (e.g., the 6th row in Fig. 12).

\section{Conclusion and future work}

In this paper, we have presented a novel salient object detection method by aggregating multiple image priors. First, multiple priors and multiple distance metrics are used to construct multiple graphs. Second, fixation and boundary information are utilized to extract foreground and background seeds, respectively. Then multiple graphs and saliency seeds are integrated into an optimized multi-graph learning framework to produce foreground and background saliency maps, which can take advantage of the complementary nature of multiple graphs to handle variant scenes. The final result is generated by fusing foreground and background saliency map. We extensively evaluate our method on five public datasets with state-of-the-art methods. Experimental results demonstrate the advantage of our method in detecting objects in complex scenes. For future work, we plan to investigate other kinds of saliency cues or priors to be embedded into our method, and also explore the combination of different distance metrics and different features, which could make better use of the complementarity of multiple graphs.

\section{Acknowledgments}

This work was supported by the Shanghai Aerospace Science and Technology Innovation Fund [grand number SAST201409] and the China National Science Foundation [grand number 71301057].

[1] V. Buso, I. Gonzlez-Daz, J. Benois-Pineau, Goal-oriented top-down probabilistic visual attention model for recognition of manipulated objects in egocentric videos, Signal Process. Image Commun. 39, Part B (2015) 418-431. 


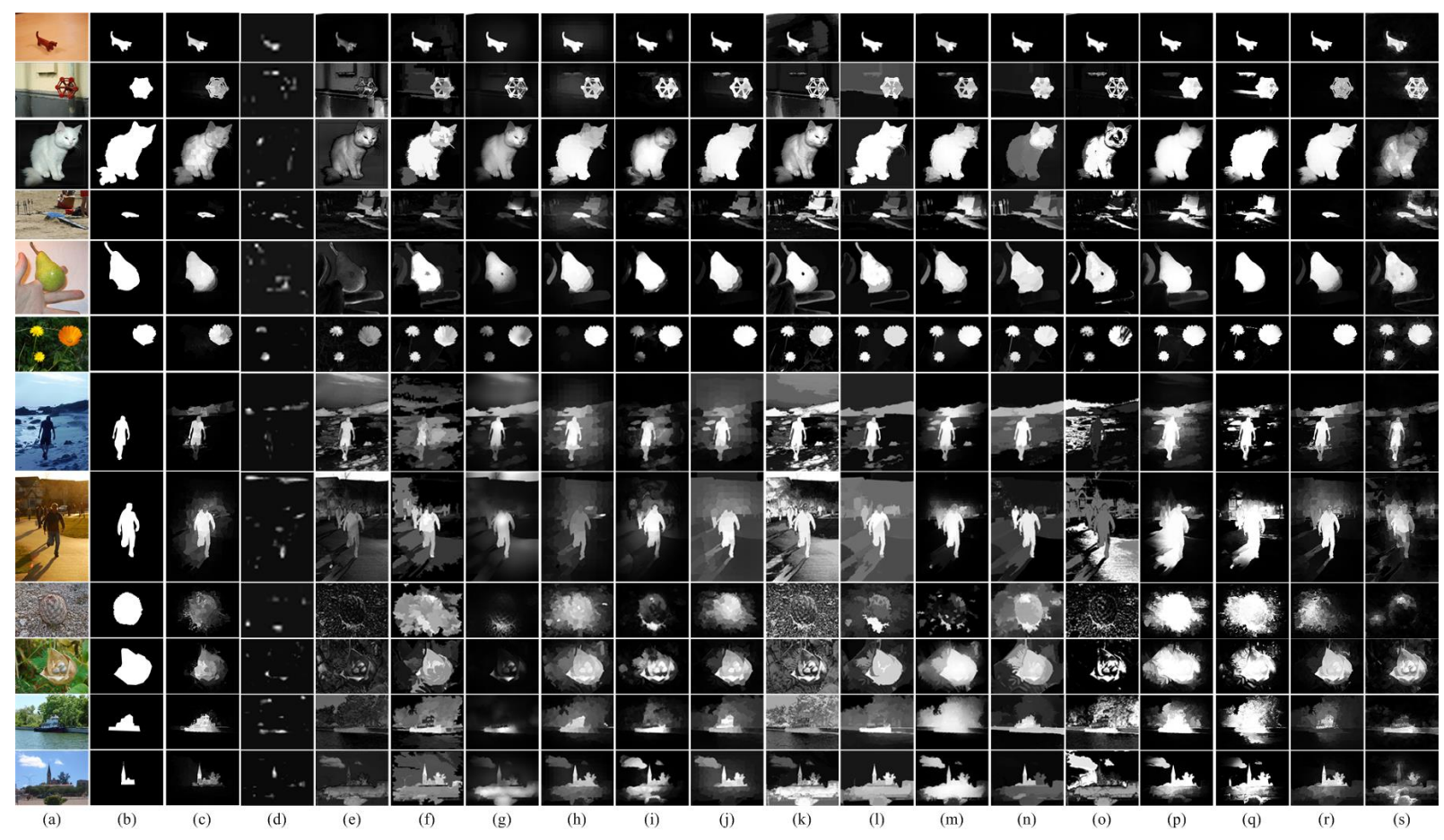

Fig. 12. Visual comparisons of our method and the other methods on five datasets. (a) original image, (b) ground truth, (c) Ours, (d) IT [8], (e) FT [54], (f) RC [14], (g) SF [50], (h) MC [15], (i) DSR [16], (j) GMR [11], (k) HC [14], (l) HS [52], (m) RBD [17], (n) ST [18], (o) GC [55], (p) MB+ [56], (q) MST [57], (r) LPS [51], (s) HDCT [24].

[2] I. Kostavelis, L. Nalpantidis, A. Gasteratos, Object recognition using saliency maps and htm learning, in: 2012 IEEE International Conference on Imaging Systems and Techniques Proceedings, 2012, pp. 528-532.

[3] A. S. Danko, S. Lyu, Better together: Fusing visual saliency methods for retrieving perceptually-similar images, in: 2015 IEEE International Conference on Consumer Electronics (ICCE), IEEE, 2015, pp. 507-508.

[4] F. Shafieyan, N. Karimi, B. Mirmahboub, S. Samavi, S. Shirani, Image retargeting using depth assisted saliency map, Signal Process. Image Commun. 50 (2017) 34-43.

[5] J. Li, X. Qian, K. Lan, P. Qi, A. Sharma, Improved image gps location estimation by mining salient features, Signal Process. Image Commun. 38 (2015) 141-150.

[6] B. Schauerte, R. Stiefelhagen, Look at this! learning to guide visual saliency in human-robot interaction, in: International Conference on Intelligent Robots and Systems, IEEE, 2014, pp. 995-1002.

[7] G. Schillaci, S. BodiroÅa, V. V. Hafner, Evaluating the effect of saliency detection and attention manipulation in human-robot interaction, International Journal of Social Robotics 5 (1) (2013) 139-152.

[8] L. Itti, C. Koch, E. Niebur, A model of saliency-based visual attention for rapid scene analysis, IEEE Trans. Pattern Anal. Mach. Intell. 20 (11) (1998) 1254-1259.

[9] T. Liu, J. Sun, N. N. Zheng, X. Tang, H. Y. Shum, Learning to detect a salient object, in: IEEE Conference on Computer Vision and Pattern Recognition, 2007, pp. 596-603.

[10] R. Achanta, F. Estrada, P. Wils, S. Ssstrunk, Salient region detection and segmentation, in: International conference on computer vision systems, Springer, 2008, pp. 66-75.

[11] C. Yang, L. H. Zhang, H. C. Lu, X. Ruan, M. H. Yang, Saliency detection via graph-based manifold ranking, in: IEEE conference on computer vision and pattern recognition, 2013, pp. 3166-3173.

[12] J. Wang, H. Lu, X. Li, N. Tong, W. Liu, Saliency detection via background and foreground seed selection, Neurocomputing 152 (2015) 359-368.

[13] A. Borji, M. M. Cheng, H. Jiang, J. Li, Salient object detection: A benchmark, IEEE Trans. Image Process. 24 (12) (2015) $5706-5722$.

[14] M. M. Cheng, N. J. Mitra, X. Huang, P. H. Torr, S. M. Hu, Global contrast based salient region detection, IEEE Trans. Pattern Anal. Mach. Intell. 37 (3) (2015) 569-82.

[15] B. W. Jiang, L. H. Zhang, H. C. Lu, C. Yang, M. H. Yang, Saliency detection via absorbing markov chain, in: IEEE International Conference on Computer Vision, 2013, pp. 1665-1672.

[16] X. H. Li, H. C. Lu, L. H. Zhang, X. Ruan, M. H. Yang, Saliency detection via dense and sparse reconstruction, in: IEEE International Conference on Computer Vision, 2013, pp. 2976-2983.

[17] W. Zhu, S. Liang, Y. Wei, J. Sun, Saliency optimization from robust background detection, in: IEEE Conference on Computer Vision and 
Pattern Recognition, 2014, pp. 2814-2821.

[18] Z. Liu, W. Zou, O. Le Meur, Saliency tree: A novel saliency detection framework, IEEE Trans. Image Process. 23 (5) (2014) $1937-1952$.

[19] I. Hwang, S. H. Lee, J. S. Park, N. I. Cho, Saliency detection based on seed propagation in a multilayer graph, Multimedia Tools and Applications (2016) 1-19.

[20] A. Borji, L. Itti, State-of-the-art in visual attention modeling, IEEE Trans. Pattern Anal. Mach. Intell. 35 (1) (2013) 185-207.

[21] A. Borji, M.-M. Cheng, H. Jiang, J. Li, Salient object detection: A survey, arXiv preprint arXiv:1411.5878 (2014).

[22] J. Li, Y. H. Tian, L. Y. Duan, T. J. Huang, Estimating visual saliency through single image optimization, IEEE Signal. Proc. Let. 20 (9) (2013) 845-848.

[23] K. Fu, C. Gong, J. Yang, Y. Zhou, I. Y.-H. Gu, Superpixel based color contrast and color distribution driven salient object detection, Signal Process. Image Commun. 28 (2013) 1448-1463.

[24] K. Jiwhan, H. Dongyoon, T. Yu-Wing, K. Junmo, Salient region detection via high-dimensional color transform and local spatial support, IEEE Trans. Image Process. 25 (1) (2016) 9-23.

[25] L. Zhu, D. A. Klein, S. Frintrop, Z. Cao, A. B. Cremers, A multisize superpixel approach for salient object detection based on multivariate normal distribution estimation, IEEE Trans. Image Process. 23 (12) (2014) 5094-5107.

[26] Z. Liu, X. Zhang, S. H. Luo, O. Le Meur, Superpixel-based spatiotemporal saliency detection, IEEE Trans. Circuits Syst. Video Technol. 24 (2014) $1522-1540$

[27] K. Fu, C. Gong, I. Gu, J. Yang, Normalized cut-based saliency detection by adaptive multi-level region merging, IEEE Trans. Image Process. 24 (12) (2015) 5671-5683.

[28] D. A. Klein, S. Frintrop, Center-surround divergence of feature statistics for salient object detection, in: 2011 Ieee International Conference on Computer Vision (ICCV), 2011, pp. 2214-2219.

[29] Y. M. Fang, Z. Z. Chen, W. S. Lin, C. W. Lin, Saliency detection in the compressed domain for adaptive image retargeting, Ieee Transactions on Image Processing 21 (2012) 3888-3901.

[30] Y. H. Tian, J. Li, S. Yu, T. J. Huang, Learning complementary saliency priors for foreground object segmentation in complex scenes, Int. J. Comput. Vision 111 (2015) 153-170.

[31] Y. Qiu, X. Sun, M. F. She, Saliency detection using hierarchical manifold learning, Neurocomputing 168 (2015) 538-549.

[32] Y. Li, X. Hou, C. Koch, J. M. Rehg, A. L. Yuille, The secrets of salient object segmentation, in: IEEE Conference on Computer Vision and Pattern Recognition, 2014, pp. 280-287.

[33] L. Vincent, P. Soille, Watersheds in digital spaces: an efficient algorithm based on immersion simulations, IEEE Trans. Pattern Anal. Mach. Intell. 13 (6) (1991) 583-598.

[34] J. Shi, J. Malik, Normalized cuts and image segmentation, IEEE Trans. Pattern Anal. Mach. Intell. 22 (8) (2000) 888-905.

[35] D. Comaniciu, P. Meer, Mean shift: A robust approach toward feature space analysis, IEEE Trans. Pattern Anal. Mach. Intell. 24 (5) (2002) 603-619.

[36] A. Levinshtein, A. Stere, K. N. Kutulakos, D. J. Fleet, S. J. Dickinson, K. Siddiqi, Turbopixels: Fast superpixels using geometric flows, IEEE Trans. Pattern Anal. Mach. Intell. 31 (12) (2009) 2290-2297.

[37] R. Achanta, A. Shaji, K. Smith, A. Lucchi, P. Fua, S. Susstrunk, Slic superpixels compared to state-of-the-art superpixel methods, IEEE Trans. Pattern Anal. Mach. Intell. 34 (11) (2012) 2274-82.

[38] Y. Xie, H. Lu, M. H. Yang, Bayesian saliency via low and mid level cues, IEEE Trans Image Process 22 (2013) 1689-98.

[39] P. Dollr, C. L. Zitnick, Structured forests for fast edge detection, in: Proceedings of the IEEE International Conference on Computer Vision, 2013, pp. 1841-1848.

[40] D. A. Klein, S. Frintrop, Salient pattern detection using w2 on multivariate normal distributions, in: Joint DAGM (German Association for Pattern Recognition) and OAGM Symposium, Springer, 2012, pp. 246-255.

[41] H. Z. Jiang, J. D. Wang, Z. J. Yuan, Y. Wu, N. N. Zheng, S. P. Li, Salient object detection: a discriminative regional feature integration approach, in: IEEE Conference on Computer Vision and Pattern Recognition, 2013, pp. 2083-2090.

[42] C. R. Givens, R. M. Shortt, A class of wasserstein metrics for probability distributions, The Michigan Mathematical Journal 31 (1984) 231-240.

[43] S. S. Zhang, C. Bauckhage, D. A. Klein, A. B. Cremers, Exploring human vision driven features for pedestrian detection, IEEE Trans. Circuits Syst. Video Technol. 25 (2015) 1709-1720. Cs8si.

[44] P. Arbelaez, M. Maire, C. Fowlkes, J. Malik, Contour detection and hierarchical image segmentation, IEEE Trans. Pattern Anal. Mach. Intell. 33 (2011) 898-916.

[45] Y. C. Wei, F. Wen, W. J. Zhu, J. Sun, Geodesic saliency using background priors, in: European Conference on Computer Vision, Springer Berlin Heidelberg, 2012, pp. 29-42.

[46] A. Borji, What is a salient object? a dataset and a baseline model for salient object detection, IEEE Trans. Image Process. 24 (2) (2015) $742-56$.

[47] J. Zhang, S. Sclaroff, Saliency detection: A boolean map approach, in: Proceedings of the IEEE International Conference on Computer Vision, 2013, pp. 153-160.

[48] D. Zhou, O. Bousquet, T. N. Lal, J. Weston, B. Scholkopf, Learning with local and global consistency, in: Advances in neural information processing systems, 2004, pp. 321-328.

[49] M. Wang, X.-S. Hua, X. Yuan, Y. Song, L.-R. Dai, Optimizing multi-graph learning: towards a unified video annotation scheme, in: Proceedings of the 15th ACM international conference on Multimedia, ACM, 2007, pp. 862-871.

[50] F. Perazzi, P. Krahenbuhl, Y. Pritch, A. Hornung, Saliency filters: Contrast based filtering for salient region detection, in: IEEE Conference on Computer Vision And Pattern Recognition, 2012, pp. 733-740.

[51] H. Li, H. Lu, Z. Lin, X. Shen, B. Price, Inner and inter label propagation: Salient object detection in the wild, IEEE Trans. Image Process. 24 (2015) 3176-3186.

[52] Q. Yan, L. Xu, J. P. Shi, J. Y. Jia, Hierarchical saliency detection, in: IEEE Conference on Computer Vision and Pattern Recognition, IEEE, 2013, pp. 1155-1162. 
[53] T. Judd, K. Ehinger, F. Durand, A. Torralba, Learning to predict where humans look, in: IEEE International Conference on Computer Vision, 2009, pp. 2106-2113.

[54] R. Achanta, S. Hemami, F. Estrada, S. Susstrunk, Frequency-tuned salient region detection, in: IEEE Conference on Computer Vision And Pattern Recognition, 2009, pp. 1597-1604.

[55] M. M. Cheng, J. Warrell, W. Y. Lin, S. Zheng, V. Vineet, N. Crook, Efficient salient region detection with soft image abstraction, in: IEEE International Conference on Computer Vision, 2013, pp. 1529-1536.

[56] J. Zhang, S. Sclaroff, Z. Lin, X. Shen, B. Price, R. Mech, Minimum barrier salient object detection at 80 fps, in: Proceedings of the IEEE International Conference on Computer Vision, 2015, pp. 1404-1412.

[57] W.-C. Tu, S. He, Q. Yang, S.-Y. Chien, Real-time salient object detection with a minimum spanning tree, in: Proceedings of the IEEE Conference on Computer Vision and Pattern Recognition, 2016, pp. 2334-2342.

[58] S. Fang, J. Li, Y. Tian, T. Huang, X. Chen, Learning discriminative subspaces on random contrasts for image saliency analysis, IEEE Trans. Neural Networks Learn. Syst. (2016).

[59] J. Li, L. Y. Duan, X. W. Chen, T. J. Huang, Y. H. Tian, Finding the secret of image saliency in the frequency domain, IEEE Trans. Pattern Anal. Mach. Intell. 37 (2015) 2428-2440.

[60] Y. Lu, W. Zhang, H. Lu, X. Y. Xue, Salient object detection using concavity context, in: IEEE International Conference on Computer Vision, 2011, pp. 233-240. 